\title{
ERRATUM
}

Sadataka Furui

\section{Propagator of the Lattice Domain Wall Fermion and the Staggered Fermion}

Published online: 24 April 2009

(C) Springer-Verlag 2009

\section{Erratum to: Few-Body Syst (2009) 45:51-63 \\ DOI 10.1007/s00601-009-0008-9}

The scale of the apscissa of Fig. 1 is in error. The momentum $p$ should be multiplied by a factor of $\frac{1}{2}$. In Table $1, c$ and $\Lambda$ of $\mathrm{DWF}_{01}, \mathrm{DWF}_{02}$ and $\mathrm{DWF}_{03}$ should be multiplied by a factor of 2 and $\frac{1}{2}$, respectively. The differences of the mass function between the staggered fermion and DWF are small.

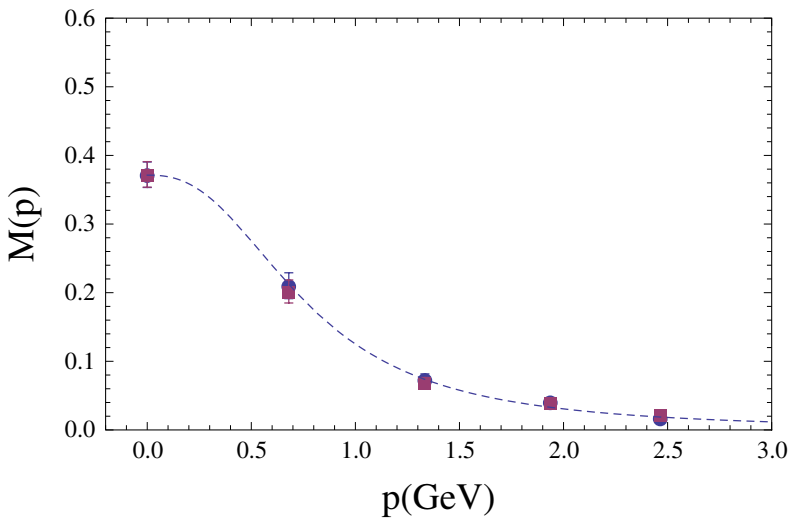

Fig. 1 The mass function of the domain wall fermion as a function of the modulus of Euclidean four momentum $p \cdot m_{f}=0.01$. (149 samples). Blue disks are $m_{L}$ (left handed quark) and red boxes are $m_{R}$ (right handed quark)

The online version of the original article can be found under doi:10.1007/s00601-009-0008-9.

S. Furui

School of Science and Engineering, Teikyo University, 1-1 Toyosatodai, Utsunomiya 320-8551, Japan

E-mail: furui@umb.teikyo-u.ac.jp 
Table 1 The fitted parameters of mass function of DWF (RBC/UKQCD) and staggered fermion (MILC)

\begin{tabular}{|c|c|c|c|c|c|}
\hline & $m_{u d} / a$ & $m_{s} / a$ & $c$ & $\Lambda(\mathrm{GeV})$ & $\alpha$ \\
\hline$\overline{\mathrm{DWF}_{01}}$ & 0.01 & 0.04 & 0.49 & $0.76(2)$ & 1.25 \\
\hline $\mathrm{DWF}_{02}$ & 0.02 & 0.04 & 0.48 & $0.80(3)$ & 1.25 \\
\hline $\mathrm{DWF}_{03}$ & 0.03 & 0.04 & 0.61 & $0.66(2)$ & 1.25 \\
\hline MILC $_{f 1}$ & 0.006 & 0.031 & 0.45 & $0.82(2)$ & 1.00 \\
\hline $\operatorname{MILC}_{f 2}$ & 0.012 & 0.031 & 0.43 & 0.89 (2) & 1.00 \\
\hline
\end{tabular}

